\title{
Intermuscular Lipoma in the Posterior Triangle of the Neck
}

\author{
Jung Sik Choi, Hyun Ho Han, Jong Won Rhie \\ Department of Plastic and Reconstructive Surgery, College of Medicine, The Catholic University of Korea, Seoul, Korea
}

No potential conflict of interest relevant to this article was reported.

Lipomas occur in $2 \%$ of general population and make up almost half of all soft tissue tumors. Intramuscular lipomas account for $0 \%$ to $5.0 \%$ of all benign adipocytic tumors, with intermuscular lipomas accounting $0.3 \%$ to $1.9 \%$ [1]. The authors present an interesting case of an intermuscular lipoma found deep within the posterior triangle of the neck.

A 49-year-old man presented with a mass that had been slowly growing in the neck over the past year. The patient did not recall any significant trauma to the area, with unremarkable medical history. Physical examination revealed a soft $5 \times 3 \mathrm{~cm}$ mass that was fixed in the left posterior triangle of the neck. A computed tomography scan revealed a hypodense $6.0 \times 5.0 \mathrm{~cm}$ mass in the intermuscular space of the left neck, bordered by splenius capitis muscle, levator scapulae muscle, and semispinalis muscle (Fig. 1). The patient wished for and consented to an exploratory operation.

Under general anesthesia, a linear skin incision was made in the posterior triangle along the relaxed skin tension line. The mass was exposed by dividing the deep cervical fascia and the levator scapulae muscle, while avoiding damage to the greater auricular nerve and spinal accessory nerve. With adequate exposure, the lesion was elevated along the natural plane of dissection and excised from the deep space (Fig. 2).

\section{Correspondence: Jong Won Rhie}

Department of Plastic and Reconstructive Surgery, Seoul St. Mary's Hospital, College of Medicine, The Catholic University of Korea, 222 Banpo-daero, Seocho-gu, Seoul 06591, Korea

E-mail: rhie@catholic.ac.kr

Received March 18, 2015 / Revised April 3, 2015 / Accepted August 4, 2015
The wound was closed in layers. The neck was dressed with compressive material to minimize the risk of seroma or hematoma formation. Patient was instructed to limit neck motion until the outpatient clinic visit. At follow-up visit one week after operation, the wound was healing well. There were no signs or symptoms of fluid collection, and the patient was allowed free active range of motion.

Histopathologic examination revealed the lesion to be consistent with lipoma (Figs. 3, 4). The patient was satisfied with the re-

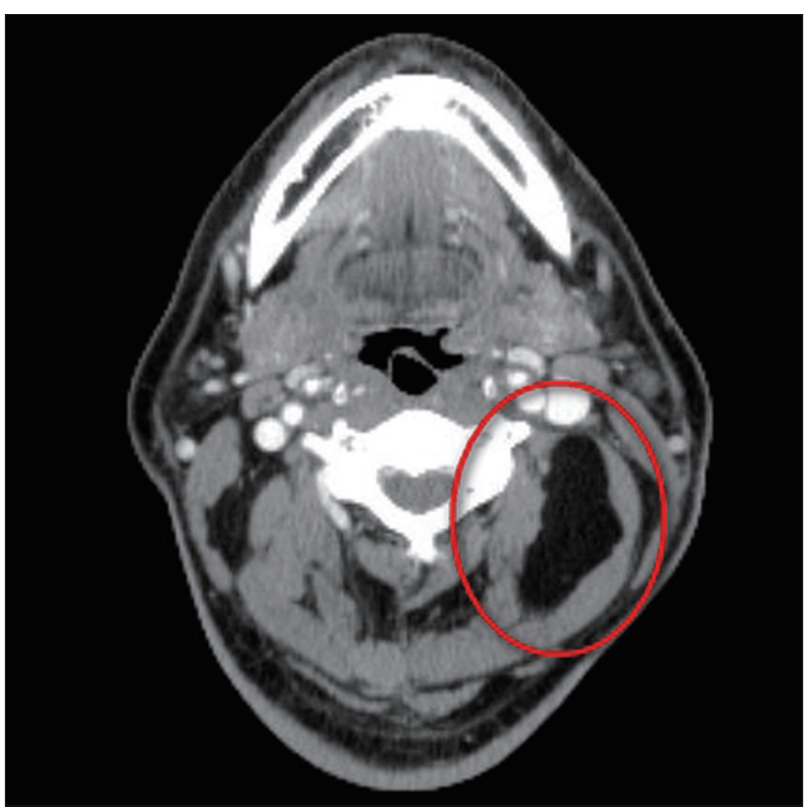

Fig. 1. Preoperative computed tomography image. A hypodense $60 \times 50 \mathrm{~mm}$ mass (red circle) is bordered by splenius capitis, levator scapulae, and semispinalis muscles. 
sult and did not experience any complication or recurrence during the 13 months of follow-up (Fig. 5).

Although lipomas are one of the most common soft tissue tumors, only few cases of deep neck lipoma have been described, and literature review showed no previously reported cases of intermuscular lipoma in the posterior triangle [2,3]. Indications for excision of deep lipoma include histologically-proven lipomas greater than $5 \mathrm{~cm}$, subfascial location, rapidly growing tumor, symptoms such as pain or firmness, and uncertain needle biopsy result [4]. In ad-

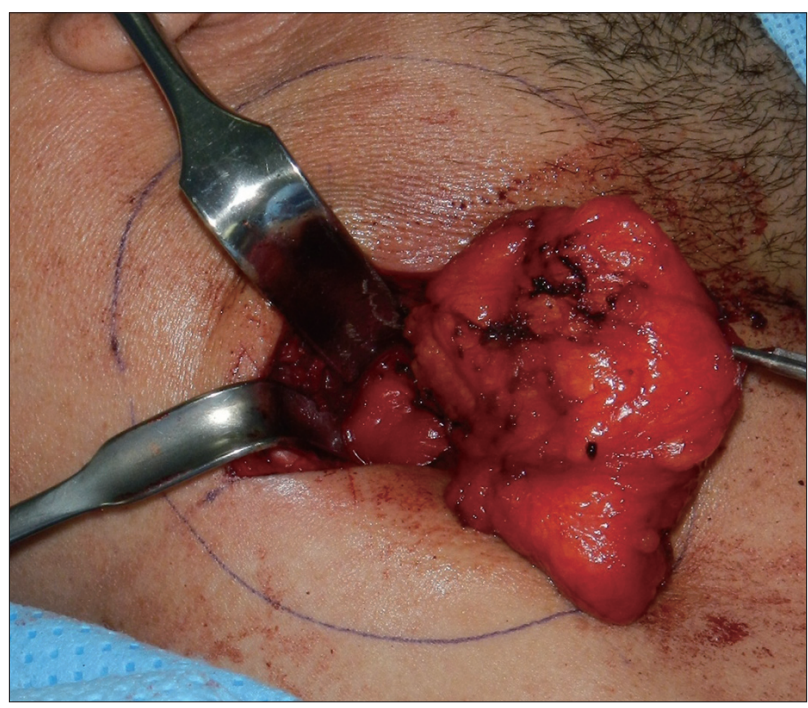

Fig. 2. Intraoperative photograph. The lipoma was delivered from deep intermuscular space after dividing the levator scapulae muscle.

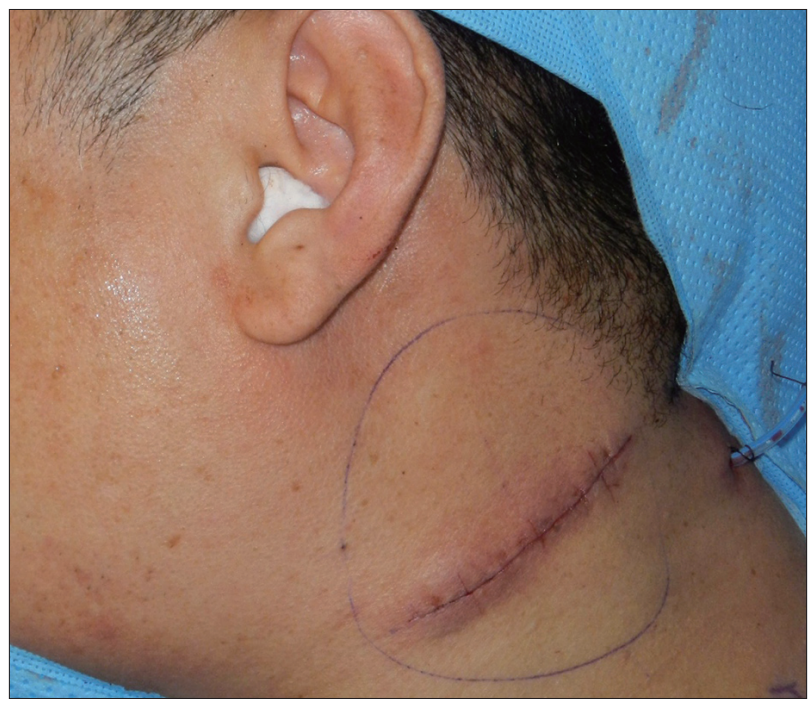

Fig. 3. Postoperative photograph. dition, surgical excision is frequently required because of the difficulty in distinguishing lipomas from other sarcomatous lesions. In our case, the intermuscular mass required complete excision because of its size and the gradual increase in size over a year. Recurrence rates for lipoma is lower for intermuscular lipoma than for intramuscular lipoma. In a study comparing intramuscular lipomas and intermuscular lipomas, 39 of 47 intramuscular lipomas were found to be infiltrative type (83\%). Recurrence rate for intramuscular and intermuscular lipomas were $16 \%$ and $0 \%$, respectively, with all of the recurrences occurring for the infiltrative intramuscular lipomas [5]. In conclusion, lipomas should be considered in the differential diagnosis of deep neck masses.

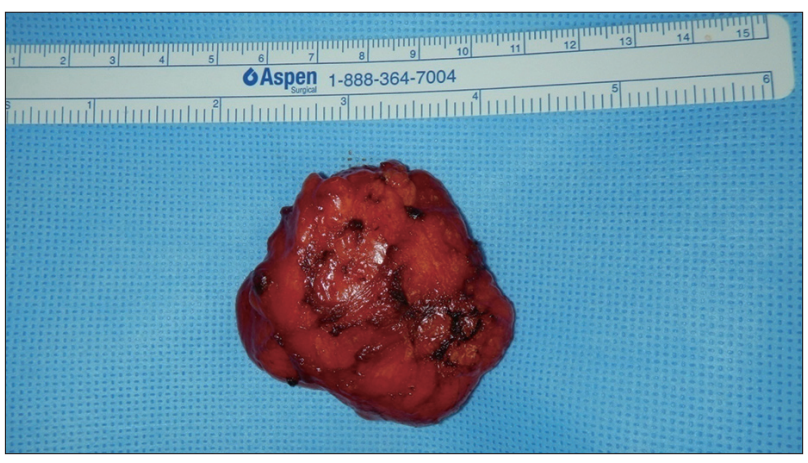

Fig. 4. Surgical specimen.

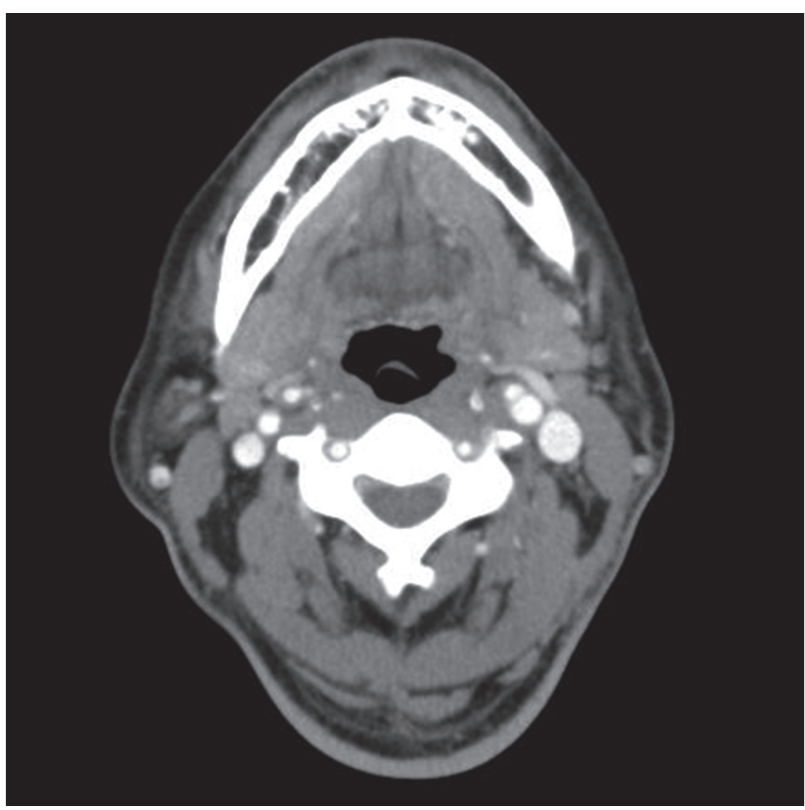

Fig. 5. Computed tomography image at 13 months after surgery. No recurrence is noted in the intermuscular space. 


\section{REFERENCES}

1. Adachi P, Kaba SP, Soubhia AM, Shinohara EH. Intermuscular lipoma of the submandibular space. Indian J Dent Res 2011;22:871-2.

2. Grecchi F, Zollino I, Candotto V, Gallo F, Rubino G, Bianchi R, et al. A case of lipoma of lateral anterior neck treated with surgical enucleation. Dent Res J (Isfahan) 2012;9:S225-8.
3. Lee HK, Hwang SB, Chung GH, Hong KH, Jang KY. Retropharyngeal spindle cell/pleomorphic lipoma. Korean J Radiol 2013;14:493-6.

4. Kaeser MA, Smith LW, Kettner NW. A case report of an intermuscular lipoma: presentation, pathophysiology, differential diagnosis. J Chiropr Med 2010;9:127-31.

5. Fletcher CD, Martin-Bates E. Intramuscular and intermuscular lipoma: neglected diagnoses. Histopathology 1988;12:275-87. 